\title{
New aspects of the C5a receptor
}

\author{
Hiroshi Nishiura, Kiyoshi Ohura \\ Department of Pharmacology, Osaka Dental University, Hirakata, Japan \\ Email: nishiu-h@cc.osaka-dent.ac.jp
}

Received 19 November 2013; revised 22 December 2013; accepted 4 January 2014

Copyright (c) 2014 Hiroshi Nishiura, Kiyoshi Ohura. This is an open access article distributed under the Creative Commons Attribution License, which permits unrestricted use, distribution, and reproduction in any medium, provided the original work is properly cited. In accordance of the Creative Commons Attribution License all Copyrights (C) 2014 are reserved for SCIRP and the owner of the intellectual property Hiroshi Nishiura, Kiyoshi Ohura. All Copyright (C) 2014 are guarded by law and by SCIRP as a guardian.

\section{ABSTRACT}

The process of apoptotic cell death for maintenance of cell homeostasis is now believed to be flexible. To examine the mechanism for this flexibility, the process of programmed cell death is sometimes divided into three phases: initiation, effector and execution. We have demonstrated that apoptotic cells commonly express a de novo synthesized C5a receptor (C5aR), which belongs to the $G$ protein-coupled receptor (GPCR) family. A natural agnostic ligand of the C5aR, C5a, is produced from plasma $\mathrm{C} 5$ by $\mathrm{C} 5$ convertase in the early phase of acute inflammation. Although it is not realistic, we found that $\mathrm{C} 5$ a can adjust apoptotic cell lifespan long. We recently have read interesting reports that apoptotic cells can release natural agnostic ligands at the initiation phase and corresponding GPCRs are already expressed on cell surfaces of apoptotic cells. Conversely, we found that apoptotic cells commonly release an alternative antagonistic/agnostic ligand of the de novo synthesized C5aR, ribosomal protein S19 (RP S19) polymer. The RP S19 polymer can adjust apoptotic cell lifespan short. Importantly, the $\mathrm{C} 5 \mathrm{a}$-dependent regulation is limited by the C5aR sensitization, but the RP S19 polymer-dependent regulation is unlimited by the C5aR desensitization. Therefore, we suggested that apoptotic cells commonly release agnostic ligands in the initiation phase that should lengthen intermittently a period of the initiation phase. Next, apoptotic cells commonly release antagonistic/agnostic ligands in the effector phase that should continue shortening a period of the effector phase. In addition, we know that an inherited erythroblastopenia is associated with mutations in the RP S19 gene. However, the roles of RP S19 in the formation of erythroblast-macrophage islands are not clearly understood. We recently have found that a different arm that the RP S19 polymer has connects the de novo synthesized C5aR on erythroblasts and the generally expressed C5aR on macrophages. Therefore, we suggested that apoptotic cells commonly release antagonistic/agnostic ligands in the execution phase that should continue connecting apoptotic cells and macrophages in the execution phase for shortening a period of the execution phase. In this review, we introduce new aspects of the C5aR in apoptotic cells and discuss the effects of the long lifespan of apoptotic cell-like neutrophils on the development of periodontitis.

\section{KEYWORDS}

Adhesion; Apoptosis; C5a Receptor; Differentiation; G Protein-Coupled Receptor; Neutrophils;

Periodontitis; Ribosomal Protein S19

\section{INTRODUCTION}

To maintain homeostasis, the fate of every cell is determined by programmed cell death [1]. Although macrophages mainly clear apoptotic cells before apoptotic cells can become secondarily necrotic, the progression of apoptosis remains unclear. We do not pathologically observe neutrophil migration into apoptotic cells. For the purpose of understanding the apoptotic process, programmed cell death can be divided into three phases: initiation, effector, and execution [2].

Surprisingly, apoptotic cells release chemoattractants such as fractalkine and sphingosine- 1 phosphate and the nucleotides ATP and uridine triphosphate during the initiation phase for apoptotic cell clearance by macrophages at the execution phase [3-5]. These chemoattractants are called find-me signals. We suggested another role of find-me signals in the initiation phase. Their receptors are commonly coupled with $\mathrm{G} \alpha \mathrm{i} \beta \gamma$ protein (GiPCRs) and/or G $\alpha q \beta \gamma$ protein (GqPCRs), which are classical but not pure chemoattractant receptors [6]. Classical chemoattractant receptors commonly mediate chemotaxis 
and secretion of at least macrophages and neutrophils, signaling through downstream extracellular signal-regulated kinase 1/2 (ERK1/2), and leading to an enhancement in cytoplasmic calcium mobilization. In contrast, pure chemoattractant receptors specifically mediate the chemotaxis of macrophages, signaling through an unknown downstream signal without an enhancement in cytoplasmic calcium mobilization. These effects suggest the presence of neutrophil-specific chemotaxis inhibitory molecules during the initiation phase.

To examine the roles of macrophages in the execution phase of apoptosis, we often consult the roles of macrophages in acute inflammation. The process of acute inflammation is sometimes divided into two phases, inflammation and resolution [7]. Lactoferrin, which is stored in primary granules inside neutrophils, commonly functions as an anti-microbial molecule during the inflammation phase by binding to lipopolysaccharides in the membrane of gram-negative bacteria [8]. Interestingly, the infiltrating neutrophils, which are classified as apoptotic cells, appear to synthesize lactoferrin de novo during the resolution phase, and the released lactoferrin functions in an autocrine manner as a neutrophil-specific chemotaxis inhibitory molecule [9]. Thus, lactoferrin appears to be one of the neutrophil-specific chemotaxis inhibitory molecules in both the inflammation and resolution phases of acute inflammation. For apoptotic cells, however, further examination will be required regarding monocyte-specific chemoattractants or neutrophil-specific chemotaxis inhibitory molecules during the initiation phase of apoptosis.

Lactoferrin released from neutrophils is also an antiapoptotic signaling molecule functioning in an autocrine manner [10]. Thus, until now, it was thought that while GPCR agonists were associated with pro-chemotaxis and anti-apoptosis, GPCR antagonists were associated with anti-chemotaxis and pro-apoptosis. However, lactoferrin is both anti-chemotactic and anti-apoptotic. A fourth molecule demonstrating pro-chemotaxis and pro-apoptosis has yet to be discovered.

It is reasonable to believe that a long lifespan is an advantage for neutrophils in destroying invasive agents or particles during the inflammatory phase of acute inflammation. In addition, GPCR ligands reportedly can regulate and lengthen the lifespan of apoptotic cells [11]. GPCRs on macrophages for find-me signals are already expressed on apoptotic cells during the initiation phase. Therefore, we suggest that find-me signals may work as accumulation (un-spread) factors and/or regulators to lengthen the lifespan of apoptotic cells and neutrophils. Apoptotic cells and neutrophils need time to present apoptotic cues for macrophages on the cell surface.

We have demonstrated that apoptotic cell lifespan is partially regulated by a balance between survival signals emanating from GiPCRs and death signals coming from death receptors [12]. There are interesting reports that constitutively active GPCRs spontaneously change their conformations from inactive forms to active forms when stimulated by factors in their microenvironment [13]. The survival signals derived from constitutively active GPCRs without ligands would be weaker than those derived from GPCRs with ligands. However, a decrement in the survival signal from constitutively active GPCRs is not sufficient to shorten the lifespan of apoptotic cells including neutrophils. Therefore, we suggest that apoptotic cells decrease their own lifespan in an autocrine manner by de novo synthesis of GiPCRs and/or GiPCR antagonists (Figure 1). Apoptotic cells must be cleared by macrophages before presenting inflammatory cues to the cell surface.

Before becoming secondarily necrotic, apoptotic cells timely express adhesion molecules for bridging with macrophages to enhance the clearance efficiency of these macrophages. Apoptotic cells expose phosphatidylserine, or macrophages release milk fat globule-EGF factor 8 protein and expose alpha(v)beta(3) integrin [14]. Milk fat globule-EGF factor 8 can bind to apoptotic cells and macrophages via phosphatidylserine and alpha(v)beta(3) integrin, respectively. The up-regulated intercellular adhesion molecule 3 on apoptotic neutrophils was recently found to directly bind with alpha(L)beta(2) integrin on macrophages [15].

We collected synovial tissue from patients with rheu-

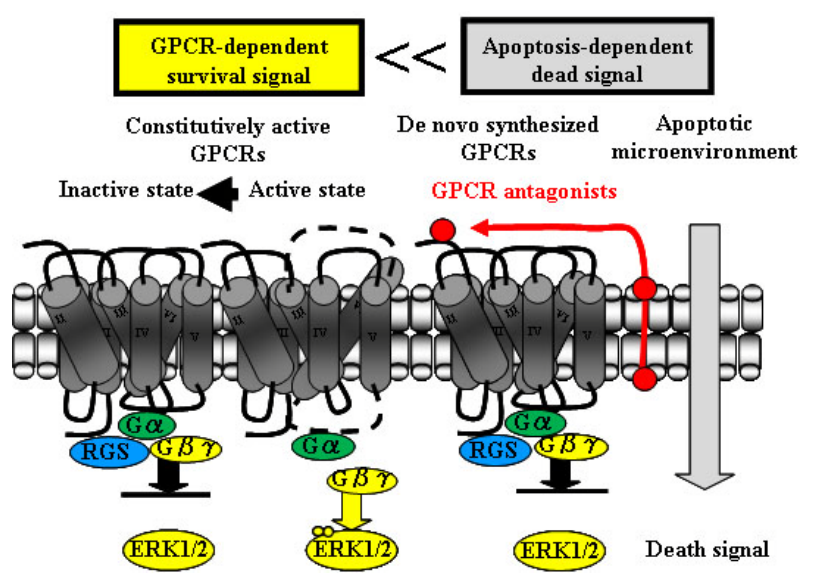

Figure 1. Pro-apoptosis during the late phase of apoptosis. When the microenvironment becomes apoptotic, cells express GPCRs and generate GPCR antagonistic ligands. These antagonists are released from the apoptotic cells and bind to their GPCRs in an autocrine manner. The GPCR-mediated signal transduction pathway induces RGS production. RGS immediately stops $G$ protein-dependent ERK1/2 phosphorylation by an association of $\mathrm{G} \alpha$ and $\mathrm{G} \beta \gamma$ subunits with not only the de novo synthesized GPCRs but also the constitutively active GPCRs. The intensity of the resulting apoptosis-dependent death signal is stronger than that of the GPCR-dependent survival signal. 
matoid arthritis and found a predominant pathological monocyte accumulation [16]. To elucidate the mechanism for this accumulation, we have studied monocytespecific chemoattractants for approximately 20 years. We previously purified the RP S19 polymer in rheumatoid arthritis-synovial tissue extracts [17]. Recently, we found that apoptotic cells commonly express the C5aR and generate the alternative C5aR ligand RP S19 polymer during the effector phase of apoptosis [18]. In the present review, we introduce new aspects of the C5aR in apoptotic cells and neutrophils and discuss the effects of the long lifespan of neutrophils on the development of periodontitis, similar to rheumatoid arthritis.

\section{THE C5AR}

The promoter region of the mouse $\mathrm{C} 5 \mathrm{aR}$ gene possesses a CCAAT sequence that can be activated by the binding of nuclear factor Y (NF-Y) [19]. NF-Y recruits RNA polymerase II and induces transcription of CCAAT boxcontaining genes. We recently found the CCAAT-box in the promoter region of the human $\mathrm{C} 5 \mathrm{aR}$ gene and the $\mathrm{C} 5 \mathrm{aR}$ protein expressed in human promyelocytic leukemia HL-60 cells, human pancreatic cancer AsPC-1 cells, and mouse fibroblastic NIH3T3 cells at $12 \mathrm{hr}$ after inducing apoptosis by using heat-shock, $\mathrm{MnCl}_{2}$, and thapsigargin, respectively [12]. Therefore, we demonstrated that the de novo synthesis of C5aR is a common event in apoptotic cells, even though cells do not constantly express the C5aR gene.

The C5aR gene does not have splice variant mRNAs. This means that C5aR expresses the same structure on all cell types. We know that C5a is generally cleaved from $\mathrm{C} 5$, which is a plasma protein released from hepatocytes, by C5 convertase (C4b2a3b and C3b3bBb). C5a functions as a C5aR agonist in the inflammatory phase of acute inflammation. We observed an anti-inflammatory phenomenon in C5 deficient mice [20]. Therefore, we believe that the $\mathrm{C} 5 \mathrm{aR}$ transmits a pro-inflammatory signal. In contrast, we observed a pro-inflammatory phenomenon in C5aR deficient mice [21]. These data suggest that the C5aR uses an alternative ligand to transmit anti-inflammatory signals.

\subsection{The Classical C5aR Ligand}

Based on the NMR structure of C5a and experiments mutating the C5aR [22], (the crystal structure of C5aR has not been produced yet), we suggest that the N-terminal positive charge cluster of $\mathrm{C} 5 \mathrm{a}-\mathrm{His}_{15}, \mathrm{Asp}_{24}, \mathrm{Cys}_{27}$, $\operatorname{Arg}_{40}, \operatorname{Arg}_{46}$, and $\mathrm{Ser}_{66}$-near the three disulfide bonds between $\mathrm{Cys}_{21}-\mathrm{Cys}_{47}, \mathrm{Cys}_{22}-\mathrm{Cys}_{54}$, and $\mathrm{Cys}_{34}-\mathrm{Cys}_{55}$ first binds to the $\mathrm{N}$-terminal negative charge cluster of C5aR-Asp 15 -Asp-Lys-Asp ${ }_{18}$. Then, the C-terminus of C5a, Leu $_{72}$-Gly-Arg ${ }_{74}$, binds to $\operatorname{Arg}_{175}$, Glu 199 , Arg $_{206}$, or
$\mathrm{Asp}_{282}$ for induction of the conformational change [23, 24]. Cook's and Hagemann's groups discuss the possibility of an interaction of a homo-polymer formation not only with the C5aR but also with the C5aR ligand [25]. The idea may lead to a correct answer about various signal transduction pathways mediated by the C5aR.

In addition, we think that the distance between the first and second binding moieties is important for C5a binding to the $\mathrm{C} 5 \mathrm{aR}$ in the purposed two-step binding theory. We re-modeled the solution structure of C5a (MMDB ID: 73492, PDB ID: 1CFA) utilizing the Molecular Operating Environment software system [26]. The resulting $22.6 \AA$ distance from $\mathrm{His}_{15}$ to $\mathrm{Gly}_{73}$ may be the key to discovering the correct binding structure of $\mathrm{C} 5 \mathrm{aR}$.

\subsection{The Classical Signal Transduction Pathway for the C5aR}

When the GDP form of the Gai2 subunit is exchanged with the GTP form via the guanidine exchange factor activity of $\mathrm{C} 5 \mathrm{aR}$, the $\mathrm{G} \beta \gamma$ subunits commonly interact with phospholipase $\mathrm{C} \beta 2$ (PLC $\beta 2)$ at the EF hand domain $\left(\mathrm{Ile}_{141}\right.$-Lys $\left._{176}\right)$ in a calcium-dependent manner for converting phosphatidylinositol 4,5-bisphosphate into inositol 1,4,5-triphosphates (IP3) and diacylglycerol [27]. However, the source for the calcium interacting with PLC $\beta 2$ is still unclear. For the P2Y (GqPCR) ligand ATP, which is released from apoptotic cells at the initiation phase, a source of calcium is the extracellular calcium entering the cell through the ligand-gated ion channels P2Xs [3]. After the binding of IP3 to the IP3 receptor expressed on the endoplasmic reticulum, intracellular calcium is released, and the calcium-released activation of the calcium channel expressed on the plasma membrane is then opened for activation of protein kinase $\mathrm{C}$ [28]. Both the phosphatidylinositol 3-kinase signal and the ERK1/2 signal are transmitted through the small GTP binding protein Ras and the MAPK kinase Raf. Meanwhile, the cytoplasmic phospholipase A2 (cPLA2) activated by extracellular calcium through calcium-released activation of the calcium channel hydrolyses arachidonic acid for an extension of the cytoplasmic calcium oscillation induced by the extracellular calcium (Figure 2 left).

\section{AN ALTERNATIVE C5AR LIGAND}

RP S19 is cross-linked between Lys $_{122}$ and $\mathrm{Gln}_{137}$ by type II tissue transglutaminase (TGII) in apoptotic cells at the effector phase of apoptosis and by activated coagulation factor XIII on platelets at the resolution phase of acute inflammation [29]. The RP S19 polymer-induced chemotaxis is inhibited by a C5aR antagonistic peptide [18, 30,31]. Based on experiments mutating RP S19, we found that an N-terminal positive charge cluster, $\mathrm{Lys}_{38^{-}}$ $\mathrm{Lys}_{43}$, is the first binding moiety and $\mathrm{Leu}_{131}-\mathrm{Asp}-\mathrm{Arg}_{133}$ is 

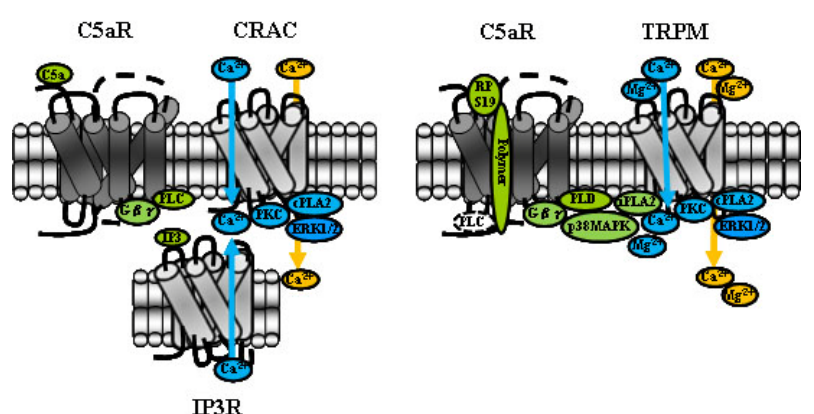

Figure 2. The C5aR-mediated cytoplasmic calcium oscillation. Left: The C5a-induced C5aR-mediated cytoplasmic calcium oscillation is extended by both the intracellular calcium through the inositol 1,4,5-triphosphate receptor (IP3R) and the extracellular calcium through calcium-released activation of the calcium channel (CRAC). Right: The RP S19 polymerinduced C5aR-mediated cytoplasmic calcium oscillation is extended by the extracellular calcium through calcium and/or magnesium channels (TRPM).

the second binding moiety. Although the RP S19 monomer does not cross-link with the anti-C5a antibody and never binds to the C5aR, the RP S19 polymer reacts with the anti-C5a antibody in western blot analysis and competitively inhibits the binding of ${ }^{125}$ I-labeled C5a to C5aR [30]. We constructed a tertiary structural model of RP S19 base on the tertiary structural data of RP S19 from Archaebacterium by utilizing the Molecular Operating Environment software system and by studying the crystal structure of RPS19 obtained from Pyrococcus abyssi $[26,32]$. We determined that the distance between the first and second binding moieties of the RP S19 molecule is $35.71 \AA$. However, the distance from $\mathrm{His}_{52}$ on one RP S19 molecule to Asp $\mathrm{A}_{132}$ on another RP S19 molecule in the RP S19 polymer is adjusted to be $22.6 \AA$. These results demonstrate that the RP S19 polymer is the alternative C5aR ligand.

Important questions remain regarding when and where the RP S19 monomer is cross-linked by TGII to predominantly attract macrophages. Human promyelocytic leukemia HL-60 cells and human pancreatic cancer AsPC- 1 cells increase TGII activity at $12 \mathrm{hr}$ after the induction of apoptosis by heat-shock [33,34]. In the same experimental setting, the monocyte chemotactic activity in each culture supernatant is increased at $24 \mathrm{hrs}$. Moreover, we recently demonstrated the production of the RP S19 polymer in apoptotic cells and a release of the RP S19 polymer in culture supernatants by western blot analysis with the anti-C5a antibody [12,35].

The amino acid sequence for $\mathrm{C} 5 \mathrm{a}$ is distinct from that of RP S19 in that RP S19 has 12 additional amino acid residues on the C-terminus (Figure 3). We prepared recombinant C5a, RP S19 polymer and C-terminus deficient RP S19 polymer [36] and found that although the RP S19 polymer attracts macrophages but not neutro-

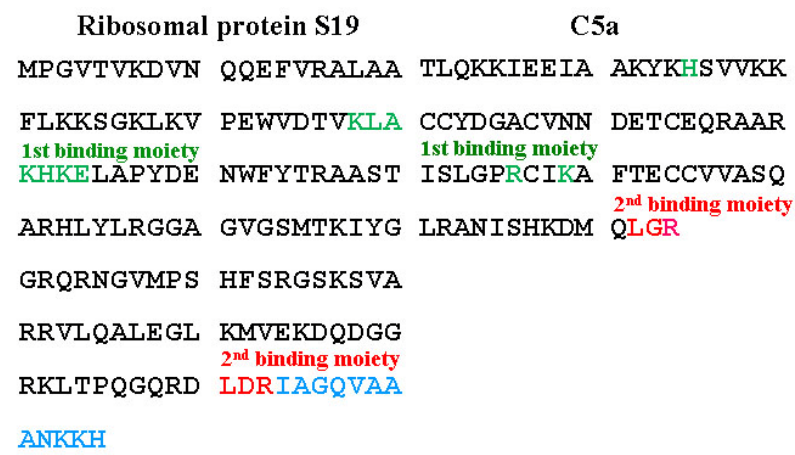

Figure 3. Distinct amino acid sequences for RP S19 and C5a. After the $\mathrm{Lys}_{38}-\mathrm{Lys}_{43}$ moiety of RP S19 binds to the C5aR, the $\mathrm{Leu}_{131}$-Asp-Arg moiety enters the active pocket of the C5aR. The Ile $_{134}-\mathrm{His}_{145}$ switches the RP S19 polymer-dependent function from agonistic to antagonistic.

phils, the C-terminus deficient RP S19 polymer attracts both macrophages and neutrophils with a chemotactic potency similar to C5a. These results were validated by using guinea pig skin because human C5a and RP S19 polymer can bind to the guinea pig C5aR [37]. In addition, we confirmed these results using the C5a peptide, Tyr-Ser-Phe-Lys-Asp-Met-Gln-Leu-Gly-Arg, the RP S19 peptide, Ac-Gly-Gln-Arg-Asp-Leu-Asp-Arg-Ile-Ala-GlyGln-Val-Ala-Ala-Ala-Asn-Lys-Lys, and the C-terminusdeficient RP S19 peptide, Ac-Gly-Gln-Arg-Asp-LeuAsp-Arg-Ile-Ala-Gly-Gln-Val-Ala-Ala-Ala-Asn. Interestingly, the results obtained by using these peptides demonstrate that although the RP S19 monomer protein does not bind to the C5aR, the RP S19 peptide attracts macrophages but not neutrophils. Thus, we strongly suggest that the distance between the first and second binding moieties of RP S19 to the C5aR plays a major role in the two-step binding theory. We also prepared recombinant C5a and C5a/RP S19, which is the 12 amino acids on the C-terminus of RP S19 connected to the C-terminus of the Gly73Asp-mutant C5a [38]. Although C5a attracts both macrophages and neutrophils, C5a/RP S19 attracts only macrophages. These results were validated by using guinea pig skin. Further study will be needed to elucidate the roles of the RP S19 polymer in the effector phase of apoptosis.

\subsection{An Alternative Signal Transduction Pathway Mediated by the Monocyte C5aR}

The RP S19 polymer binds to the monocyte C5aR and shifts a $\mathrm{G} \beta \gamma$ subunit-dependent downstream signal from ERK1/2 pathway to p38 mitogen-activated protein kinase (p38MAPK) pathway independent of intracellular (endoplasmic reticulum) calcium release [39]. The p38MAPK signal is artificially produced by C5a stimulation following simultaneous treatment with phosphatidylinositol 3-kinase and PLC inhibitors (LY294002 and 
U73122) at concentrations equal to half of their respective $\mathrm{IC}_{50}$ values. We also determined that the RP S19 C-terminus interacts with the Gla domain of prothrombin at low calcium concentrations [40]. These data indicate that the RP S19 C-terminal Lys $_{143}$ Lys $_{144}$ moiety acts as a competitor against calcium for the activation of PLC $\beta 2$ in macrophages. There is an interesting report that phospholipase D (PLD) downstream of the interaction of the $\mathrm{G} \beta \gamma$ subunits with ADP-ribosylation factor exchange factors not only catalyzes the hydrolysis of phosphatidylcholine to phosphatidic acid and choline but also drives the p38MAPK pathway [41,42]. We found that the RP S19 polymer-induced p38MAPK phosphorylation upstream of both alternative extracellular calcium oscillation and cell migration was inhibited by the PLD inhibitor 5-fluoro-2-indolyl des-chlorohalopemide (unpublished data), indicating a participation of PLD in the G $\beta \gamma$ subunit-dependent p38MAPK pathway mediated by the monocyte C5aR (Figure 2 right). Importantly, C5aR internalization is not required in the PLD-dependent pathway. Further studies will be required to determine the mechanism for the monocyte C5aR ligand-specific selection of second messengers.

It has been reported that a reciprocal activation between calcium-independent PLA2 (iPLA2) and cPLA2 at the front side of cells affects the maximum potency of cell migration [27]. We confirmed that a non-specific PLA2 inhibitor is efficient for creating cytoplasmic calcium oscillation by extracellular calcium through extracellular calcium and/or magnesium channels including the transient receptor potential melastatin 7 channels in human mast HMC-1 cells, human peripheral blood macrophages and HL-60-derived macrophage-like cells [18]. The ready state of iPLA2 bound by calmodulin at hydrophobic anchor residues $\left(\mathrm{Val}_{691}-\mathrm{Phe}_{709}\right)$ is altered to the active state via the replacement of calcium from the EF hand domains [43]. The ready state of the transient receptor potential melastatin 7 channel is also maintained at least in part by the binding of calmodulin to the hydrophobic anchor residues of $\mathrm{Met}_{1}-\mathrm{Arg}_{14}$ [44,45]. Again, these data indicate the presence of a specific ligand-gated ion channel for the C5aR ligands.

\subsection{An Alternative Signal Transduction Pathway Mediated by the Neutrophil C5aR}

The C-terminus of RP S19 binds in an autocrine manner to a cell type-specific molecule in addition to the C5aR generally expressed on neutrophils and the C5aR synthesized de novo on apoptotic cells [12]. The complex of the RP S19 C-terminus with the cell type-specific molecule blocks the interaction between the G $\alpha \mathrm{i} \beta \gamma$ protein and any second messenger. However, C5a can partially delay the apoptotic process in neutrophils via an activation of the
ERK1/2 pathway [46]. In contrast, we showed that the RP S19 polymer promotes the apoptotic process in apoptotic cells $[12,35]$. The RP S19 polymer induces the de novo synthesis of the regulator of $G$ protein signaling 3 (RGS3) during the effector phase of apoptosis. The apoptotic process is extended by neutralizing the RP S19 polymer with an anti-RP S19 antibody or by blocking the apoptotic C5aRs with C5aR antagonistic peptides. The ERK1/2 signal in HL-60 cells is strengthened in an RGS3 knockdown and weakened by RGS3 overexpression. Moreover, the regulation of RGS3 expression is efficient in HL-60 cells and inefficient in human monocytic cancer THP-1 cells. The HL-60 cells or THP-1 cells can differentiate into neutrophil-like cells or monocytelike cells by treatment with dimethyl sulfoxide or phorbol 12-myristate 13-acetate, respectively. Therefore, we suggest that the RP S19 polymer-dependent pro-apoptotic signal is specifically increased in apoptotic cells including neutrophils. The interaction of the apoptotic cell type-specific molecule with the RP S19 polymer likely induces the generation of RGS3 (Figure 4). We hypothesize that the RP S19 polymer plays a role in forming apoptotic cell-macrophage islands unlimited by C5aR internalization that initiate efficient processing of apoptotic cell clearance without any inflammatory cues as part of the cell maintenance mechanism.

\subsection{An Alternative Signal Transduction Pathway Mediated by the Erythroblast C5aR}

In a recently reported case of Diamond black-fan anemia, an inherited erythroblastopenia was associated with mutations in the RP S19 gene [47]. Matsson et al. suggested a role for the constitutive pro-apoptotic signal through a

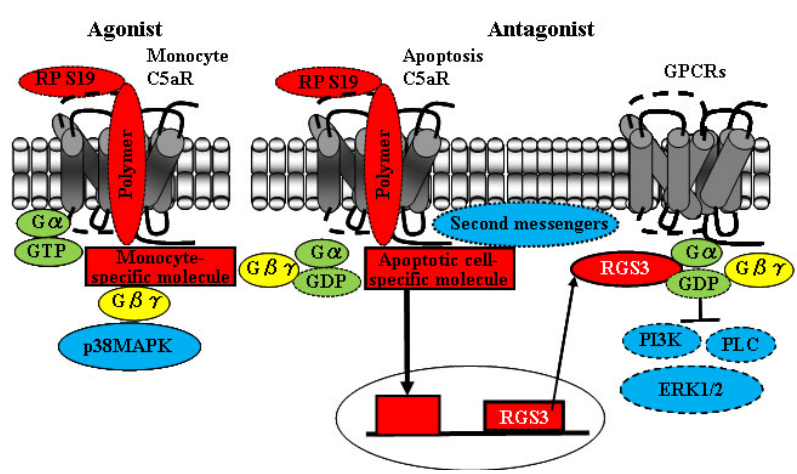

Figure 4. The RP S19 C-terminus-dependent duel agonistic and antagonistic mechanisms. Agonist; an RP S19 polymer is bound to a monocyte $\mathrm{C} 5 \mathrm{aR}$, and the $\mathrm{Gi}$ protein interaction with second messengers is partially blocked by the RP S19 C-terminus, resulting in signal transduction mediated through the p38 MAPK pathway. Antagonist; an RP S19 polymer is bound to a neutrophil $\mathrm{C} 5 \mathrm{aR}$, and the Gi protein interaction with second messengers is completely blocked by the RP S19 C-terminus-apoptotic cell-specific molecule complex. The apoptotic cell-specific molecule promotes RGS3 expression. 
defect in the ribosome formation mediated by the mutant RP S19. However, the roles of the erythroblast stagespecific activation of pro-apoptotic signals and the formation of erythroblast-macrophage islands under normal conditions are not clearly understood. Recently, we found that human erythroleukemia K562 cells synthesize C5aRs de novo and release RP S19 polymers during hemin-induced erythropoiesis [48]. The K562 cell differentiation is stopped before the formation of erythroblastlike cell-macrophage islands by either neutralization of the RP S19 polymer with an anti-RP S19 antibody or blockade of the C5aR with C5aR antagonistic peptides. These data indicate that the RP S19 polymer plays a role in forming erythroblast-macrophage islands via a reciprocal expression of C5aRs unlimited by C5aR internalization that ensures efficient differentiation and apoptotic cell clearance without any inflammatory cue as part of the cell maintenance mechanism. To validate our new idea, we are now developing RP S19 polymer-dependent functionally deficient mice.

\section{CHEMOTACTIC GIPCRS IN APOPTOSIS}

We previously examined the CCAAT sequence at $-75 \mathrm{bp}$ in the promoter region of the C5aR gene (NM_001736.3) that is bound by the apoptosis-induced activated transcription factor NF-Y. We demonstrated that the apoptotic cell lifespan is shortened by the RP S19 polymer-dependent generation of RGS3, which decreases ERK1/2 phosphorylation via de novo synthesized GiPCRs through re-association of the $\mathrm{G} \alpha \mathrm{i}$ and $\mathrm{G} \beta \gamma$ subunits of the $\mathrm{G} \alpha \mathrm{i} \beta \gamma$ protein (Figure 4). To confirm our hypothesis, we searched for CCAAT sequences in the fMet-Leu-Phe (fMLP) receptor $(F P R)$ gene and the leukotriene B4 (LTB4) receptor $(B L T)$ gene.

In addition to FPR1, there are two other receptor subtypes, FPR2 and FPR3 [49]. The function of FPR2 on the plasma membrane is similar to the prototypical receptor FPR1. Trp-Lys-Tyr-Met-Val-Met is a selective agonist of FPR2, which is an acetylated amino-terminal peptide derived from the cleavage of the human heme-binding protein [50]. The coupling of the FPR2 with G $\alpha \mathrm{i} \beta \gamma$ protein is also similar to that of the FPR1. However, the downstream signal pathways for the FPR2 remain a mystery, indicating the need to find or develop additional FPR2 ligands. An interesting study reported that the N-terminus of annexin 1 ( Ala $\left._{2}-\mathrm{Lys}_{26}\right)$ functions as an FPR antagonist in the effector phase of apoptosis in neutrophils [51]. The FPR3 binds the same FPR2 ligand; however, the FPR3 is coupled with the G $\alpha 16 \beta \gamma$ protein and localized on small intracellular vesicles. We found that CCAAT sequences are located at $-548 \mathrm{bp},-859 \mathrm{bp}$, and -524 bp in the promoter region of the FPR1 gene (NM_002029.3), FPR2 gene (NM_001462.3), and FPR3 gene (NM_002030.3), respectively. The distance between a binding sequence of a transcription factor and a start codon is inversely related to the intensity of the gene expression. Therefore, further study is needed examining the relationship between FPR and pro-apoptosis.

The BLT receptor is divided into two subtypes, BLT1 and BLT2 [52]. Their homology is $45.2 \%$. The affinity of LTB4 is 20 times lower than that of LTB1 for the BLT2 receptor subtype. In contrast to the BLT1 receptor, the BLT2 receptor appears to activate at least three $\mathrm{G} \alpha$ subunits, G $\alpha \mathrm{i}, \mathrm{G} \alpha \mathrm{q}$-like, and G $\alpha$ z. The loose selection mechanism of the BLT2 receptor for the $\mathrm{G} \alpha \beta \gamma$ proteins makes it difficult to recognize the roles of this receptor subtype in inflammation and apoptosis. We found that the CCAAT sequence is located at -143 bp or -54 bp in the promoter regions of the BLT1 gene (NM_181657.3) or BLT2 gene (NM_019839.4), respectively. We also determined that the pro-apoptotic potency of HL-60 cells induced by $\mathrm{MnCl}_{2}$ is partially suppressed by the simultaneous presence of LTB4 (unpublished data). We suggest that apoptotic cells express BLT receptors and release an unknown BLT antagonist in an autocrine manner for promoting pro-apoptosis, as previously shown for the C5aR.

\section{THE C5AR IN PERIODONTITIS}

Distinct pathological findings such as continuously acute inflammation in articular cavities and chronic inflammation in synovium are observed in patients with rheumatoid arthritis [53]. Similarly, continuously acute inflammation in periodontal pockets and chronic inflammation in junctional epithelium and periodontal membranes are observed in patients with periodontitis. The gram-negative bacteroides such as Porphyromonas gingivalis, Tannerella forsythia and Aggregatibacter actinomycetemcomitans have long been studied as a potential cause of periodontitis [54,55]. Bacteroides are commonly found not only in periodontal pockets but also in the junctional epithelium where they directly digest fibroblasts of the junctional epithelium and the periodontal membranes by an enzymatic function of collagenases. Importantly, lipopolysaccharide, which is a membrane component of the gram-negative bacteroides, induces the production of several types of cytokines in macrophages, such as tumor necrosis factor $\alpha$, interferon $\alpha / \beta$, interleukin-6 and interleukin-17, that shift acute inflammation to chronic inflammation. We have discussed in depth the macrophage-modified mechanisms for development of chronic inflammation. In contrast to macrophages, excessive activation of the infiltrating neutrophils is found in periodontal pockets [56]. We have not fully discussed the neutrophil-modified mechanisms for the change from acute to chronic inflammation.

Therefore, in this section of the review we examine 
published reports to study the potential effects of bacteroides on the neutrophil C5aR. Porphyromonas gingivalis generates an arginine-specific cysteine proteinase and a lysine-specific cysteine proteinase. The arginine-specific cysteine proteinase cleaves an N-terminal C5a fragment from plasma C5 [57]. Wingrove et al. suggested that there is a C5a-dependent predominant infiltration of neutrophils into periodontal pockets. The lysine-specific cysteine proteinase cleaves an $\mathrm{N}$-terminal fragment of the $\mathrm{C}_{5} \mathrm{aR}, \mathrm{Pro}_{9}-\mathrm{Thr}_{29}$ [58]. The cleaved fragment contains an Asp $_{15}$-Asp-Lys-Asp 18 moiety, which is the first binding site of C5a on the C5aR. We think that the RP S19 polymer but not C5a can function in the periodontal pocket. However, the binding affinity of the neutrophil $\mathrm{C} 5 \mathrm{aR}$ in the circulation is lower than that in the periodontal pocket, resulting in a weak apoptotic signal induced by the RP S19 polymer and offering one reason why the neutrophils infiltrating the periodontal pocket gain excessive activity.

In addition, Tannerella forsythia generates a metalloproteinases karilysin and prevents classical and lectin two complement systems by the efficient degradation of mannose-binding lectin, ficolin-2, ficolin-3 and C4 [59]. Interestingly, karilysin cleaves C5a from plasma C5. Jusko et al. focus on the C5a-induced migration of neutrophils. In contrast, we suggest a cleavage of the neutrophil C5aRs in the gingival pocket by Tannerella forsythia, as previously shown for Porphyromonas gingivalis [58].

Aggregatibacter actinomycetemcomitans generates two exotoxins, a cytolethal distending toxin and a leukotoxin [60]. The cytolethal distending toxin directly promotes cell cycle arrest at both the G1 and G2/M phases in gingival fibroblasts and periodontal ligament cells [61]. Leukotoxin, a large pore-forming protein that belongs to the Repeat in Toxin family, induces apoptosis via the lymphocyte function associated receptor 1 (LFA-1) of neutrophils. The LFA-1 functions as an adhesion molecule, binding to intercellular adhesion molecule 1 on antigen-presenting cells for promoting proliferation/differentiation of $\mathrm{T}$ cells. We are interested in the different signal transduction pathways mediated by the same LFA-1 but in a cell type-specific manner, as previously shown for the C5aR $[39,48]$. It is very complicated, because the oral manifestation appears to change with the type and quantity of bacteria in a circadian rhythm.

We developed an experimental rat model of gingivitis (ODUS/Odu) derived from the Wistar-Kyoto strain [62]. Five bacteroides, Haemophilus actinomycetemcomitans $Y-4$, Bacteroides gingivalis 381, Bact. intermedius ATCC 25261, Capnocytophaga sp. M-12 and Eikenella corrodens $O D U$, have been detected in extracts of rat dental plaque [63]. We are very interesting in the enzymatic effects of the above listed bacteroides on C5a and the neutrophil C5aR.

\section{CONCLUSIONS}

In this review, we demonstrate that apoptotic cells commonly release agnostic ligands in the initiation phase that should lengthen intermittently a period of the initiation phase via the generally expressed GPCRs in an autocrine manner. Next, apoptotic cells commonly release antagonistic/agnostic ligands in the effector phase that should continue shortening a period of the effector phase via the de novo synthesized GPCRs in an autocrine manner. The antagonistic/agnostic ligands in the execution phase should continue connecting apoptotic cells and macrophages in the execution phase via the GPCRs for shortening a period of the execution phase.

We discuss the enzymatic effects of the bacteroides in periodontitis at least on C5aR, FPR and BLT of neutrophils. The process of apoptotic cell death for maintenance of cell homeostasis should be wrong. We believe that the neutrophil C5aR is one of the therapeutic targets for periodontitis.

\section{ACKNOWLEDGEMENTS}

Hiroshi Nishiura designed the study and edited this manuscript. Kiyoshi Ohura gave me advice to write the section about the C5aR in periodontitis. Contract grant sponsor 1: the Ministry of Education, Culture, Sports, Science, and Technology; Contract grant number 1: 22590362. Contract grant sponsor 2: the Japan Science and Technology Agency; Contract grant number 2: AS242Z00056Q.

\section{REFERENCES}

[1] Nagata, S. (2010) Apoptosis and autoimmune diseases. Annals of the New York Academy of Sciences, 1209, 1016. http://dx.doi.org/10.1111/j.1749-6632.2010.05749.x

[2] Gonzalez, V.M., Fuertes, M.A., Alonso, C. and Perez J.M. (2001) Is cisplatin-induced cell death always produced by apoptosis? Molecular pharmacology, 59, 657-663.

[3] Ravichandran, K.S. (2011) Beginnings of a good apoptotic meal: The find-me and eat-me signaling pathways. Immunity, 35, 445-455. http://dx.doi.org/10.1016/j.immuni.2011.09.004

[4] White, G.E. and Greaves D.R. (2012) Fractalkine: A survivor's guide: Chemokines as antiapoptotic mediators. Arteriosclerosis, Thrombosis, and Vascular Biology, 32, 589-594.

http://dx.doi.org/10.1161/ATVBAHA.111.237412

[5] Hait, N.C., Oskeritzian, C.A., Paugh, S.W., Milstien, S. and Spiegel, S. (2006) Sphingosine kinases, sphingosine 1-phosphate, apoptosis and diseases. Biochimica et Biophysica Acta, 1758, 2016-2026. http://dx.doi.org/10.1016/j.bbamem.2006.08.007

[6] Jia, N., Semba, U., Nishiura, H, Kuniyasu, A., Nsiama, T.K., Nishino, N. and Yamamoto, T. (2010) Pivotal Ad- 
vance: Interconversion between pure chemotactic ligands and chemoattractant/secretagogue ligands of neutrophil C5a receptor by a single amino acid substitution. Journal of Leukocyte Biology, 87, 965-975.

http://dx.doi.org/10.1189/jlb.1009649

[7] Freire, M.O. and Van Dyke, T.E. (2013) Natural resolution of inflammation. Periodontology 2000, 63, 149-164. http://dx.doi.org/10.1111/prd.12034

[8] Legrand, D. (2012) Lactoferrin, a key molecule in immune and inflammatory processes. Biochemistry and Cell Biology, 90, 252-268. http://dx.doi.org/10.1139/011-056

[9] Bournazou, I., Pound, J.D., Duffin, R., Bournazos, S., Melville, L.A., Brown, S.B., Rossi, A.G. and Gregory C. D. (2009) Apoptotic human cells inhibit migration of granulocytes via release of lactoferrin. Journal of Clinical Investigation, 119, 20-32. http://dx.doi.org/10.1172/JCI36226

[10] Wong, S.H., Francis, N., Chahal, H., Raza, K., Salmon, M., Scheel-Toellner, D. and Lord J.M. (2009) Lactoferrin is a survival factor for neutrophils in rheumatoid synovial fluid. Rheumatology (Oxford), 48, 39-44. http://dx.doi.org/10.1093/rheumatology/ken412

[11] Youn, B.S., Yu, K.Y., Oh, J., Lee, J., Lee, T.H. and Broxmeyer, H.E. (2002) Role of the CC chemokine receptor 9/TECK interaction in apoptosis. Apoptosis, 7, 271-276. http://dx.doi.org/10.1023/A:1015320321511

[12] Nishiura, H., Nonaka, H., Revollo, I.S., Semba, U., Li, Y., Ota, Y., Irie, A., Harada, K., Kehrl, J.H. and Yamamoto, T. (2009) Pro- and anti-apoptotic dual functions of the $\mathrm{C} 5$ a receptor: Involvement of regulator of $\mathrm{G}$ protein signaling 3 and extracellular signal-regulated kinase. Laboratory Investigation, 89, 676-694. http://dx.doi.org/10.1038/labinvest.2009.27

[13] Milligan, G. (2003) Constitutive activity and inverse agonists of G protein-coupled receptors: A current perspective. Molecular Pharmacology, 64, 1271-1276. http://dx.doi.org/10.1124/mol.64.6.1271

[14] Miyanishi, M., Segawa, K. and Nagata, S. (2012) Synergistic effect of Tim4 and MFG-E8 null mutations on the development of autoimmunity. International Immunology, 24, 551-559. http://dx.doi.org/10.1093/intimm/dxs064

[15] Kristof, E., Zahuczky, G., Katona, K., Doro, Z., Nagy, E. and Fesus, L. (2013) Novel role of ICAM3 and LFA-1 in the clearance of apoptotic neutrophils by human macrophages. Apoptosis, 18, 1235-1251. http://dx.doi.org/10.1007/s10495-013-0873-z

[16] Nishiura, H., Tanaka, J., Takeya, M., Tsukano, M., Kambara, T. and Imamura T. (1996) IL-8/NAP-1 is the major T-cell chemoattractant in synovial tissues of rheumatoid arthritis. Clinical Immunology and Immunopathology, 80, 179-184.

[17] Nishiura, H., Shibuya, Y., Matsubara, S., Tanase, S., Kambara, T. and Yamamoto, T. (1996) Monocyte chemotactic factor in rheumatoid arthritis synovial tissue. Probably a cross-linked derivative of S19 ribosomal protein. The Journal of Biological Chemistry, 271, 878-882. http://dx.doi.org/10.1074/jbc.271.2.878

[18] Nishiura, H., Zhao, R. and Yamamoto, T. (2011) The role of the ribosomal protein S19 C-terminus in altering the chemotaxis of leukocytes by causing functional differences in the C5a receptor response. The Journal of Biochemistry. http://dx.doi.org/10.1093/jb/mvr067

[19] Hunt, J.R., Martin, C.B. and Martin, B.K. (2005) Transcriptional regulation of the murine $\mathrm{C} 5 \mathrm{a}$ receptor gene: NF-Y is required for basal and LPS induced expression in macrophages and endothelial cells. Molecular Immunology, 42, 1405-1415.

http://dx.doi.org/10.1016/j.molimm.2005.01.002

[20] Snyderman, R., Phillips, J.K. and Mergenhagen, S.E. (1971) Biological activity of complement in vivo. Role of C5 in the accumulation of polymorphonuclear leukocytes in inflammatory exudates. The Journal of Experimental medicine, 134, 1131-1143. http://dx.doi.org/10.1084/jem.134.5.1131

[21] Hopken, U.E., Lu, B., Gerard, N.P. and Gerard, C. (1997) Impaired inflammatory responses in the reverse arthus reaction through genetic deletion of the $\mathrm{C} 5 \mathrm{a}$ receptor. The Journal of Experimental Medicine, 186, 749-756. http://dx.doi.org/10.1084/jem.186.5.749

[22] Monk, P.N., Scola, A.M., Madala, P. and Fairlie, D.P. (2007) Function, structure and therapeutic potential of complement C5a receptors. British Journal of Pharmacology, 152, 429-448. http://dx.doi.org/10.1038/sj.bjp.0707332

[23] Cook, W.J., Galakatos, N., Boyar, W.C., Walter, R.L. and Ealick, S.E. (2010) Structure of human desArg-C5a. Biological Crystallography, 66, 190-197. http://dx.doi.org/10.1107/S0907444909049051

[24] Hagemann, I.S., Miller, D.L., Klco, J.M., Nikiforovich, G.V. and Baranski, T.J. (2008) Structure of the complement factor 5 a receptor-ligand complex studied by disulfide trapping and molecular modeling. The Journal of Biological Chemistry, 283, 7763-7775.

http://dx.doi.org/10.1074/jbc.M709467200

[25] Roed, S.N., Orgaard, A., Jorgensen, R. and De Meyts, P. (2012) Receptor oligomerization in family B1 of G-protein-coupled receptors: focus on BRET investigations and the link between GPCR oligomerization and binding cooperativity. Frontiers in Endocrinology (Lausanne), 3, 62. http://dx.doi.org/10.3389/fendo.2012.00062

[26] Nishiura, H., Chen, J., Ota, Y., Semba, U., Higuchi, H., Nakashima, T. and Yamamoto, T. (2010) Base of molecular mimicry between human ribosomal protein S19 dimer and human C5a anaphylatoxin. International Immunopharmacology, 10, 1541-1547. http://dx.doi.org/10.1016/j.intimp.2010.09.002

[27] Cathcart, M.K. (2009) Signal-activated phospholipase regulation of leukocyte chemotaxis. The Journal of Lipid Research, 50, S231-S236. http://dx.doi.org/10.1194/jlr.R800096-JLR200

[28] Mollapour, E., Linch, D.C. and Roberts, P.J. (2001) Activation and priming of neutrophil nicotinamide adenine dinucleotide phosphate oxidase and phospholipase A(2) are dissociated by inhibitors of the kinases p42(ERK2) and p38(SAPK) and by methyl arachidonyl fluorophosphonate, the dual inhibitor of cytosolic and calcium-independent phospholipase A(2). Blood, 97, 2469-2477. 
http://dx.doi.org/10.1182/blood.V97.8.2469

[29] Nishiura, H., Tanase, S., Sibuya, Y., Nishimura, T. and Yamamoto, T. (1999) Determination of the cross-linked residues in homo-dimerization of S19 ribosomal protein concomitant with exhibition of monocyte chemotactic activity. Laboratory Investigation, 79, 915-923.

[30] Nishiura, H., Shibuya, Y. and Yamamoto, T. (1998) S19 ribosomal protein cross-linked dimer causes monocytepredominant infiltration by means of molecular mimicry to complement C5a. Laboratory Investigation, 78, 16151623.

[31] Arumugam, T.V., Shiels, I.A., Woodruff, T.M., Reid, R.C., Fairlie, D.P. and Taylor, S.M. (2002) Protective effect of a new $\mathrm{C} 5 \mathrm{a}$ receptor antagonist against ischemia-reperfusion injury in the rat small intestine. Journal of Surgical Research, 103, 260-267. http://dx.doi.org/10.1006/jsre.2002.6369

[32] Gregory, L.A., Aguissa-Toure, A.H., Pinaud, N., Legrand, P., Gleizes, P.E. and Fribourg, S. (2007) Molecular basis of Diamond-Blackfan anemia: Structure and function analysis of RPS19. Nucleic Acids Research, 35, 5913-5921. http://dx.doi.org/10.1093/nar/gkm626

[33] Horino, K., Nishiura, H., Ohsako, T., Shibuya, Y., Hiraoka, T., Kitamura, N. and Yamamoto, T. (1998) A monocyte chemotactic factor, S19 ribosomal protein dimer, in phagocytic clearance of apoptotic cells. Laboratory Investigation, 78, 603-617.

[34] Nishimura, T., Horino, K., Nishiura, H., Shibuya, Y., Hiraoka, T., Tanase, S. and Yamamoto, T. (2001) Apoptotic cells of an epithelial cell line, AsPC-1, release monocyte chemotactic S19 ribosomal protein dimer. The Journal of Biochemistry, 129, 445-454.

http://dx.doi.org/10.1093/oxfordjournals.jbchem.a002876

[35] Nishiura, H., Tanase, S., Shibuya, Y., Futa, N., Sakamoto, T., Higginbottom, A., Monk, P., Zwirner, J. and Yamamoto, T. (2005) S19 ribosomal protein dimer augments metal-induced apoptosis in a mouse fibroblastic cell line by ligation of the C5a receptor. Journal of Cellular Biochemistry, 94, 540-553.

http://dx.doi.org/10.1002/jcb.20318

[36] Shrestha, A., Shiokawa, M., Nishimura, T., Nishiura, H., Tanaka, Y., Nishino, N., Shibuya, Y. and Yamamoto, T. (2003) Switch moiety in agonist/antagonist dual effect of S19 ribosomal protein dimer on leukocyte chemotactic C5a receptor. The American Journal of Pathology, 162, 1381-1388.

http://dx.doi.org/10.1016/S0002-9440(10)63934-X

[37] Regal, J.F. and Fraser, D.G. (1990) Recombinant human C5a-induced bronchoconstriction in the guinea-pig: A histamine independent mechanism. Pulmonary Pharmacology, 3, 79-87.

http://dx.doi.org/10.1016/0952-0600(90)90036-I

[38] Oda, Y., Tokita, K., Ota, Y., Li, Y., Taniguchi, K., Nishino, N., Takagi, K., Yamamoto, T. and Nishiura, H. (2008) Agonistic and antagonistic effects of C5a-chimera bearing S19 ribosomal protein tail portion on the C5a receptor of monocytes and neutrophils, respectively. Journal of Biochemistry, 144, 371-381.

http://dx.doi.org/10.1093/jb/mvn077
[39] Nishiura, H., Tokita, K., Li, Y., Harada, K., Woodruff, T.M., Taylor, S.M., Nsiama, T.K., Nishino, N. and Yamamoto, T. (2010) The role of the ribosomal protein S19 $\mathrm{C}$-terminus in Gi protein-dependent alternative activation of p38 MAP kinase via the C5a receptor in HMC-1 cells. Apoptosis, 15, 966-981. http://dx.doi.org/10.1007/s10495-010-0511-y

[40] Nishiura, H., Tanase, S., Tsujita, K., Sugiyama, S., Ogawa, H., Nakagaki, T., Semba, U. and Yamamoto, T. (2011) Maintenance of ribosomal protein S19 in plasma by complex formation with prothrombin. European Journal of Haematology, 86, 436-441.

http://dx.doi.org/10.1111/j.1600-0609.2011.01585.X

[41] Song, H., Wohltmann, M., Tan, M., Bao, S., Ladenson, J.H. and Turk, J. (2011) Group VIA PLA $2\left(\mathrm{iPLA}_{2} \beta\right)$ is activated upstream of p38 MAP kinase in pancreatic islet $\beta$ cell signaling. The Journal of Biological Chemistry, 287, 5528-5541. http://dx.doi.org/10.1074/jbc.M111.285114

[42] Knizhnik, A.V., Kovaleva, O.V., Komelkov, A.V., Trukhanova, L.S., Rybko, V.A., Zborovskaya, I.B. and Tchevkina, E.M. (2012) Arf6 promotes cell proliferation via the PLD-mTORC1 and p38MAPK pathways. Journal of Cellular Biochemistry, 113, 360-371. http://dx.doi.org/10.1002/jcb.23362

[43] Harteneck, C. (2005) Function and pharmacology of TRPM cation channels. Naunyn-Schmiedeberg's Archives of Pharmacology, 371, 307-314. http://dx.doi.org/10.1007/s00210-005-1034-x

[44] Jenkins, C.M., Wolf, M.J., Mancuso, D.J. and Gross, R.W. (2001) Identification of the calmodulin-binding domain of recombinant calcium-independent phospholipase $\mathrm{A}_{2} \beta$. Implications for structure and function. The Journal of Biological Chemistry, 276, 7129-7135.

http://dx.doi.org/10.1074/jbc.M010439200

[45] Mason, M.J., Schaffner, C., Floto, R.A. and Teo, Q.A. (2012) Constitutive expression of a $\mathrm{Mg}^{2+}$-inhibited $\mathrm{K}^{+}$ current and a TRPM7-like current in human erythroleukemia cells. American Journal of Physiology-Cell Physiology, 302, C853-C867. http://dx.doi.org/10.1152/ajpcell.00071.2011

[46] Perianayagam, M.C., Balakrishnan, V.S., Pereira, B.J. and Jaber, B.L. (2004) C5a delays apoptosis of human neutrophils via an extracellular signal-regulated kinase and Bad-mediated signalling pathway. European Journal of Clinical Investigation, 34, 50-56.

[47] Matsson, H., Klar, J., Draptchinskaia, N., Gustavsson, P., Carlsson, B., Bowers, D., de Bont, E. and Dahl, N. (1999) Truncating ribosomal protein S19 mutations and variable clinical expression in diamond-blackfan anemia. Human Genetics, 105, 496-500.

[48] Nishiura, H., Zhao, R. and Yamamoto, T. (2012) Dual functions of the C5a receptor as a connector for the K562 erythroblast-like cell-THP-1 macrophage-like cell island and as a sensor for the differentiation of the K562 erythroblast-like cell during haemin-induced erythropoiesis. Clinical and Developmental Immunology, 2012, Article ID: 187080. http://dx.doi.org/10.1155/2012/187080

[49] Rabiet, M.J., Macari, L., Dahlgren, C. and Boulay, F. (2011) $\mathrm{N}$-formyl peptide receptor 3 (FPR3) departs from the homo- 
logous FPR2/ALX receptor with regard to the major processes governing chemoattractant receptor regulation, expression at the cell surface, and phosphorylation. The Journal of Biological Chemistry, 286, 26718-26731. http://dx.doi.org/10.1074/jbc.M111.244590

[50] Migeotte, I., Riboldi, E., Franssen, J.D., Gregoire, F., Loison, C., Wittamer, V., Detheux, M., Robberecht, P., Costagliola, S., Vassart, G., Sozzani, S., Parmentier, M. and Communi, D. (2005) Identification and characterization of an endogenous chemotactic ligand specific for FPRL2. The Journal of Experimental Medicine, 201, 83-93. http://dx.doi.org/10.1084/jem.20041277

[51] Parente, L. and Solito, E. (2004) Annexin 1: More than an anti-phospholipase protein. Inflammation Research, 53, 125-132. http://dx.doi.org/10.1007/s00011-003-1235-Z

[52] Yokomizo, T., Kato, K., Terawaki, K., Izumi, T. and Shimizu, T. (2000) A second leukotriene B(4) receptor, BLT2. A new therapeutic target in inflammation and immunological disorders. The Journal of Experimental Medicine, 192, 421-432. http://dx.doi.org/10.1084/jem.192.3.421

[53] Agnihotri, R. and Gaur, S. (2013) Rheumatoid arthritis in the elderly and its relationship with periodontitis: A review. Geriatrics \& Gerontology International. http://dx.doi.org/10.1111/ggi.12062

[54] Wakabayashi, H., Kondo, I., Kobayashi, T., Yamauchi, K., Toida, T., Iwatsuki, K. and Yoshie, H. (2010) Periodontitis, periodontopathic bacteria and lactoferrin. Biometal: An International Journal on the Role of Metal Ions in Biology, Biochemistry, and Medicine, 23, 419-424. http://dx.doi.org/10.1007/s10534-010-9304-6

[55] Ogrendik, M. (2012) Does periodontopathic bacterial infection contribute to the etiopathogenesis of the autoimmune disease rheumatoid arthritis? Discovery Medicine, 13, 349-355

[56] Scott, D.A. and Krauss, J. (2012) Neutrophils in periodontal inflammation. Frontiers of Oral Biology, 15, 5683. http://dx.doi.org/10.1159/000329672

[57] Wingrove, J.A., DiScipio, R.G., Chen, Z., Potempa, J.,
Travis, J. and Hugli, T.E. (1992) Activation of complement components C3 and C5 by a cysteine proteinase (gingipain-1) from Porphyromonas (Bacteroides) gingivalis. The Journal of Biological Chemistry, 267, 1890218907

[58] Jagels, M.A., Ember, J.A., Travis, J., Potempa, J., Pike, R. and Hugli, T.E. (1996) Cleavage of the human C5A receptor by proteinases derived from Porphyromonas gingivalis: Cleavage of leukocyte C5a receptor. Advances in Experimental Medicine and Biology, 389, 155-164. http://dx.doi.org/10.1007/978-1-4613-0335-0_19

[59] Jusko, M., Potempa, J., Karim, A.Y., Ksiazek, M., Riesbeck, K., Garred, P., Eick, S. and Blom, A.M. (2012) A metalloproteinase karilysin present in the majority of Tannerella forsythia isolates inhibits all pathways of the complement system. Journal of Immunology, 188, 23382349. http://dx.doi.org/10.4049/jimmunol.1101240

[60] Johansson, A. (2011) Aggregatibacter actinomycetemcomitans leukotoxin: A powerful tool with capacity to cause imbalance in the host inflammatory response. Toxins, 3, 242-259. http://dx.doi.org/10.3390/toxins3030242

[61] Belibasakis, G.N., Mattsson, A., Wang, Y., Chen, C. and Johansson, A. (2004) Cell cycle arrest of human gingival fibroblasts and periodontal ligament cells by Actinobacillus actinomycetemcomitans: Involvement of the cytolethal distending toxin. APMIS: Acta Pathologica, Microbiologica, et Immunologica Scandinavica, 112, 674685.

http://dx.doi.org/10.1111/j.1600-0463.2004.apm1121006. $\underline{x}$

[62] Ohura, K., Shinohara, M., Ogata, K., Nishiyama, A. and Mori, M. (1990) Leucocyte function in rats with naturally occurring gingivitis. Archives of Oral Biology, 35, S185S187. http://dx.doi.org/10.1016/0003-9969(90)90154-3

[63] Konakajima, Y., Tani, A., Ohura, K., Shinohara, M., Ogata, K., Mori, M. and Sagawa, H. (1990) Humoral immune responses in experimental gingivitis in rats. Archives of Oral Biology, 35, S181-S183. http://dx.doi.org/10.1016/0003-9969(90)90153-2 